\title{
Stereotactic radiosurgery and hypofractionated stereotactic radiotherapy for residual or recurrent cranial base and cervical chordomas
}

\author{
Steven D. Chang, M.D., David P. Martin, M.D., Elizabeth Lee, R.N., N.P., \\ AND JOHN R. ADLER, JR., M.D. \\ Departments of Neurosurgery and Radiation Oncology, Stanford University School of Medicine, \\ Stanford, California
}

\begin{abstract}
Object. In patients with chordomas the lesions often recur. Furthermore, the location of some chordomas within the base of the skull and the cervical spine can prevent complete resection from being achieved. Previous series have shown that stereotactic radiosurgery can be used as a treatment for residual chordomas with good overall results. The authors review their experience in using linear accelerator (LINAC) stereotactic radiosurgery to treat patients with recurrent and/or residual cranial base and cervical chordomas.

Methods. Ten patients with chordomas (eight with cranial base and two with cervical lesions [below C-2]) underwent LINAC stereotactic radiosurgery. The mean patient age was 49 years (range 30-73 years). There were seven men and three women. Three patients had undergone one prior surgery, five had undergone two previous surgeries, and two had undergone three prior operations. The mean radiation dose was 19.4 Gy (range 18-24 Gy), and the maximum intratumoral dose averaged 27 Gy (range 24.1-33.1 Gy). The mean secondary collimator size was $14.4 \mathrm{~mm}$ (range 7.5-20 $\mathrm{mm}$ ). The volume of the tumor treated ranged from 1.1 to $21.5 \mathrm{ml}$. In five patients a standard frame-based LINAC radiosurgery system was used, whereas in the other five the CyberKnife, a frameless image-guided LINAC radiosurgical system, was used. All patients were available for follow-up review, which averaged 4 years (range 1-9 years). Over the course of follow up, one chordoma (10\%) was smaller in size, seven were stable, and two chordomas progressed (one in a patient who underwent reoperation and a second course of stereotactic radiosurgery, and the second in a patient who underwent reoperation alone). There were no new neurological deficits noted following radiosurgery in the eight of 10 patients in whom there was no tumor progression, and no patient developed radiation-induced necrosis.

Conclusions. Stereotactic radiosurgery can be used to treat patients with recurrent or residual chordomas with excellent tumor control rates. Longer follow-up review in larger series is warranted to confirm these findings.
\end{abstract}

KEY WORDS • chordoma • radiosurgery • cervical spine • skull base $\bullet$ stereotaxis

Chordomas arise from the intraosseous remnants of the notochord. ${ }^{12,14}$ They develop in the axial skeleton anywhere from the clivus to the sacrum, and they comprise 1 to $4 \%$ of primary bone tumors. ${ }^{23}$ Most chordomas (45$55 \%$ ) occur in the sacrococcygeal region, whereas a smaller subset occurs at the base of skull $(35 \%)$ or the vertebrae within the spine $(10-15 \%){ }^{23}$ Chordomas usually occur in a patient's fourth decade of life, but they are also found in younger and older populations. The lesions typically display a male/female sex ratio of $2: 1$, except for intracranial chordomas, which appear to be evenly distributed between the sexes. ${ }^{23}$

Chordomas grow as expansile tumors that destroy bone. When present at the base of the skull, they often infiltrate cranial nerves and other adjacent neurological structures. Because these tumors involve normal cranial base anatomy, it can be difficult to achieve a complete resection and

Abbreviations used in this paper: LINAC = linear accelerator; $\mathrm{MR}=$ magnetic resonance; $\mathrm{PFS}=$ progression-free survival. failure to do so usually results in regrowth of chordoma. ${ }^{11,12,14,33}$ In one series of 46 chordoma patients treated by an experienced skull base surgical team, ${ }^{12}$ recurrence of tumor within 5 years was demonstrated in $31 \%$ of patients. Furthermore, at least half of the patients had undergone multiple surgeries, and, as with other skull base tumors, multiple operations for recurrences can result in new neurological deficits, which ultimately affect patient quality of life. In an earlier series of 155 patients with chordoma, the authors demonstrated only a 1.5-year mean survival for patients who had undergone surgery alone compared with a 5.2-year average survival for those who had undergone surgery followed by radiotherapy. ${ }^{14}$ The overall long-term prognosis in this patient population is poor, with overall survival rates of $51 \%$ and $35 \%$ at 5 and 10 years, respectively, after subtotal surgery followed by radiotherapy. $6,9,14$

Stereotactic radiosurgery has emerged as a potential treatment for some patients with chordomas either following surgery alone or surgery and radiotherapy. The steep dose gradient achievable with radiosurgery minimizes the 
S. D. Chang, et al.
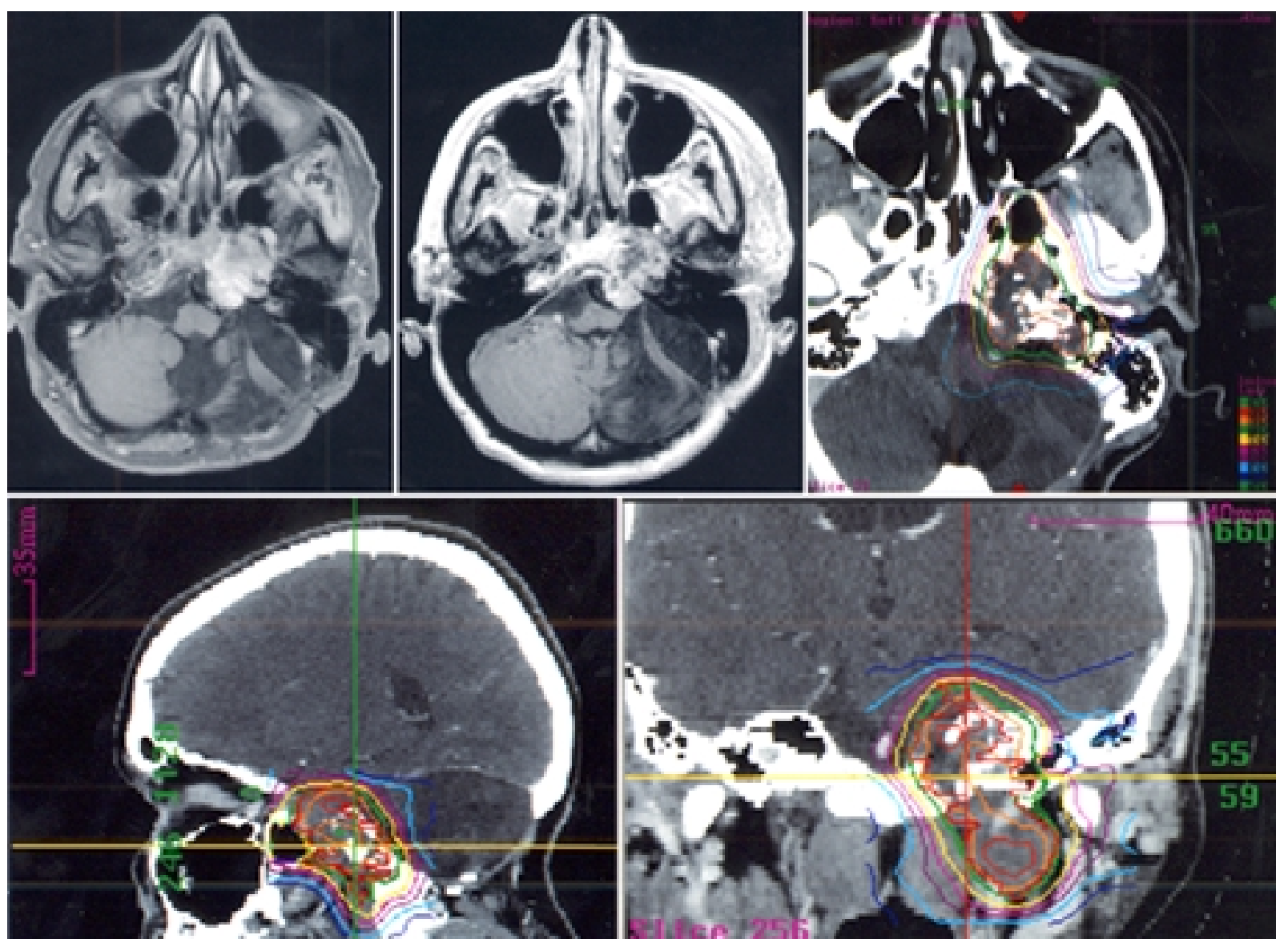

Fig. 1. Case 6. Upper Left: Axial $\mathrm{T}_{1}$-weighted MR image revealing the residual tumor in the left cavernous sinus, petrous bone, and clivus. A dose of 18.1 Gy to the $75 \%$ isodose line was used in two fractions to treat the residual volume. A secondary collimator of $17.5 \mathrm{~mm}$ was used, and the maximum intratumoral dose was $24.1 \mathrm{~Gy}$. Upper Center: Axial gadolinium-enhanced $\mathrm{T}_{1}$-weighted $\mathrm{MR}$ image obtained at 2-year follow up, demonstrating no change in the size of the tumor. The patient's treatment plan is shown in axial (upper right), coronal (lower left), and sagittal (lower right) views.

amount of radiation that is delivered outside the tumor target. As a result, it is possible to deliver a much larger, and presumably more efficacious, dose to tumor without exceeding the radiation-related tolerance of normal tissues. In addition, several authors have hypothesized that a therapeutic gain may be achieved by treating slowly proliferating tumors, such as chordomas, with larger-sized fractions, a treatment that is only possible with radiosurgical techniques. ${ }^{30,35}$ In this report, we review our experience at Stanford University in the treatment of patients with chordomas in whom LINAC stereotactic radiosurgery was performed.

\section{CLINICAL MATERIAL AND METHODS}

\section{Patient Population}

Ten patients with cranial base or cervical chordomas underwent stereotactic radiosurgery between 1993 and 2000. The mean patient age was 49 years (range 30-73 years). There were seven men and three women. Eight patients harbored cranial base chordomas centered in the clival/petroclival region (Fig. 1). Two patients harbored cervical chordomas: one based at $\mathrm{C}-4$, and the second involving C-4 and C-5 (Fig. 2). All patients had undergone at least one prior surgery, ranging from 3 weeks to 13 years prior to radiosurgical treatment. Five patients $(50 \%)$ had undergone two previous surgeries whereas two patients $(20 \%)$ had undergone three (Fig. 1).

\section{Irradiation Treatments}

Five patients underwent LINAC-based stereotactic radiosurgery in which we used a technique modeled after that of Winston and Lutz: ${ }^{34}$ computerized tomography scans were fused with MR images. Spherical treatment volumes were achieved with four noncoplanar arcs by using a 4- or 6-mV LINAC with an 80 to $100 \mathrm{~cm}$ sourceto-axis distance. The treatment dose was selected on the basis of tumor size, location, proximity to critical neurological structures, and the number of isocenters. Multiple isocenters and arc modifications were used to approximate nonspherical tumor volumes. Arcs were also modified to minimize radiation dose to the brainstem and anterior visual pathways. The treatment dose was prescribed to the $80 \%$ isodose contour at the edge of tumor. 


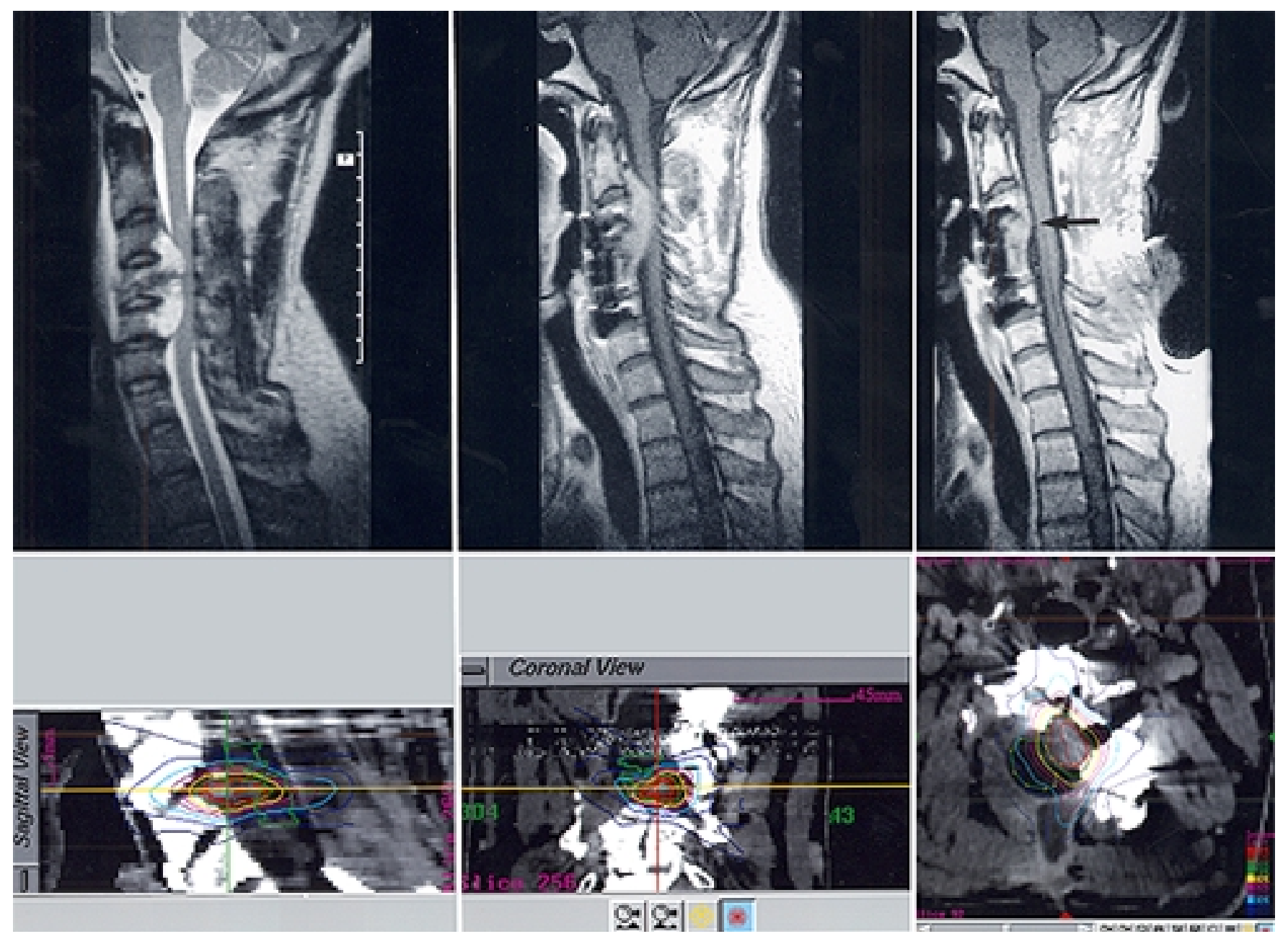

Fig. 2. Case 9. Magnetic resonance images. Upper Left: Sagittal gadolinium-enhanced $\mathrm{T}_{1}$-weighted image demonstrating a C3-5 chordoma. Upper Center: Sagittal image, obtained 10 months later, revealing recurrence centered at C2-4. Upper Right: Sagittal image, obtained 2 months after the second surgery; the recurrent chordoma (arrow) was treated with CyberKnife radiosurgery. The treatment plan shows the isodose lines in sagittal (lower left), axial (lower center), and views (lower right).

The CyberKnife (Accuray, Sunnyvale, CA) has been used recently for the treatment of brain tumors with complex shapes or locations that are difficult to treat using frame-based systems; to date, five patients with chordomas have been treated using this system. Instead of skeletal fixation for localization, target position is continually updated using x-ray image-to-image correlation. The precision of localization $(<0.3 \mathrm{~mm})$ is comparable with that which can be achieved by frames, and performance with treatment fields less than $7 \mathrm{~cm}$ is similar to much larger medical LINACs. There are several benefits of the CyberKnife: 1) precision targeting, comparable with skeletal fixation; 2) fractionated radiosurgery of tumors adjacent to critical structures, such as the anterior visual pathways; 3) improved dosimetry by using nonisocentric treatment planning for complex nonspherical treatment volumes; and 4) radiosurgery for central nervous system tumors outside the head.

The five patients with cranial base or cervical chordomas in whom the CyberKnife was used underwent con- struction of an Aquaplast mask that immobilizes the head without requiring a metal stereotactic head frame. Thinslice $(1.25-\mathrm{mm})$ contrast-enhanced computerized tomography scans were then obtained and were used alone in four of the five CyberKnife-treated patients or fused with MR images in the fifth patient for treatment planning. The CyberKnife treatment planning software allows for the use of either isocentric or, in many cases, nonisocentricbased treatment planning (Fig. 2). As with frame-based radiosurgery, the treatment dose to the tumor margin is based on volume, location, and history of fractionated radiotherapy.

The treatment dose for the 10 patients varied between $18 \mathrm{~Gy}$ and $24 \mathrm{~Gy}$ (mean $19.4 \mathrm{~Gy}$ ), which was prescribed to the 70 to $80 \%$ isodose contour at the edge of tumor. The maximum intratumoral dose ranged from 24.1 to $33.1 \mathrm{~Gy}$ (mean $27 \mathrm{~Gy}$ ). Circular secondary collimators ranged in diameter from 7.5 to $20 \mathrm{~mm}$ (mean $14.4 \mathrm{~mm}$ ). The tumor volume treated ranged from 1.1 to $21.5 \mathrm{ml}$. Four patients received single-fraction treatments. In one patient the tu- 
TABLE 1

Summary of characteristics in 10 patients with chordoma undergoing radiosurgery

\begin{tabular}{clcccc}
\hline \hline Case & $\begin{array}{c}\text { Tumor } \\
\text { Location }\end{array}$ & $\begin{array}{c}\text { No. of } \\
\text { Isocenters* }\end{array}$ & $\begin{array}{c}\text { Dose } \\
\text { (Gy) }\end{array}$ & $\begin{array}{c}\text { No. of } \\
\text { Frac- } \\
\text { tions }\end{array}$ & $\begin{array}{c}\text { Tumor } \\
\text { Margin Pre- } \\
\text { scription Dose }\end{array}$ \\
\hline 1 & clivus & 2 & 24 & 2 & $92 \%$ \\
2 & clivus & 2 & 20 & 1 & $89 \%$ \\
3 & clivus & 3 & 18 & 3 & $94 \%$ \\
4 & clivus & 1 & 18 & 1 & $93 \%$ \\
5 & clivus & 2 & 20 & 1 & $93 \%$ \\
6 & clivus & CyberKnife & 18 & 2 & $94 \%$ \\
7 & clivus & CyberKnife & 20 & 3 & $95 \%$ \\
8 & clivus & CyberKnife & 18 & 1 & $91 \%$ \\
9 & cervical spine & CyberKnife & 18 & 3 & $91 \%$ \\
10 & cervical spine & CyberKnife & 19 & 3 & $95 \%$ \\
\hline
\end{tabular}

* The CyberKnife uses a nonisocentric inverse treatment planning algorithm.

mor received two fractions (18.1 Gy to $75 \%$ isodose line) because of the larger tumor volume $(21.5 \mathrm{ml})$ and its proximity to the anterior visual structures. In both patients harboring cervical chordomas the lesions received 18 or 19 Gy to the tumor margin ( $75 \%$ isodose line) in three fractions secondary to the proximity of the spinal cord. In one patient with a clival chordoma in whom 21 Gy was delivered to the tumor margin regrowth of the tumor occurred 3 years following radiosurgery treatment; following this second subtotal resection, the patient underwent a second course of radiosurgery in three fractions of 20 Gy to a 8.5$\mathrm{ml}$ volume (Table 1).

\section{Follow-Up Course}

Patients underwent clinical follow-up examination at 3 and 6 months posttreatment and then every 6 months. Neurological status was recorded and all complications were noted. Every 6 months following radiosurgery, MR images were obtained (a minimum of $3.5-\mathrm{mm}$ slice thickness). These studies were used to follow the changes in size of treated chordoma and to assess for the presence of radiation-induced necrosis. Tumor size was calculated by obtaining direct measurements along three axes on follow-up MR images and comparing these with preatreatment MR imaging values. A tumor was considered to have decreased in size if it was reduced by $3 \mathrm{~mm}$ or greater in one or more dimensions. Tumors were considered to have increased in size if one or more dimensions increased by $3 \mathrm{~mm}$ or greater. Local neurosurgeons of some patients who lived a long distance from Stanford performed follow-up imaging and forwarded the studies to Stanford for review.

\section{RESULTS}

All patients were available for follow-up review, which averaged 4 years postradiosurgery (range 1-9 years). Based on our radiographic multidimensional measurement criteria, one chordoma was smaller in size (10\%), seven were unchanged in size, and two had progressed (one tumor harbored by a patient who underwent reoperation and a second course of radiosurgery and the other by a patient who underwent reoperation alone). In using the recently defined Response Evaluation Criteria in Solid Tumors for determining tumor response to treatment, ${ }^{13,31}$ in which a unidimensional measurement of longest tumor diameter is used, the results were unchanged: one chordoma decreased in size during follow up, seven stabilized in size, and two progressed. With respect to clinical followup examination, eight patients (80\%) experienced no new neurological symptoms postradiosurgery. The two patients in whom tumor progression developed (at 3 years and 2 years, respectively) after radiosurgery sustained new deficits. In the first patient new right-sided third and sixth crainial nerve palsies developed secondary to tumor growth within his right cavernous sinus; in the second a sixth cranial nerve palsy developed secondary to tumor progression. Despite reoperation and repeated radiosurgery in the first patient and reoperation alone in the second patient, the symptoms did not resolve. None of the preradiosurgery symptoms (that is, cranial nerve palsies) resolved in the other eight patients despite the provision of radiosurgery. No cases of radiation-induced necrosis or other radiation-induced complications were noted in this small series.

\section{ILLUSTRATIVE CASES}

\section{Case 6}

This 34-year-old woman presented in 1984 with sudden loss of consciousness due to hemorrhage of a previously undiagnosed posterior fossa tumor involving the left petrous bone and clivus (Fig. 1). The patient underwent an emergency craniotomy at an outside institution, and subtotal resection of a chordoma was achieved. Residual postoperative deficits included left-sided hearing loss, intermittent diplopia, and partial left facial palsy, and vocal cord paralysis. Follow up included serial MR imaging, and in 1992 a second craniotomy was performed via a retromastoid approach for a subtotal resection of the tumor. In 1998, serial MR imaging again demonstrated an increase in the size of the recurrent tumor $(145 \%$ increase compared with that observed in 1996), and the patient underwent a third craniotomy via a pterional approach for debulking of the tumor. At the time of surgery, a portion of the tumor was observed to be located within the cavernous sinus. Postoperatively, the residual chordoma was treated with CyberKnife stereotactic radiosurgery.

\section{Case 9}

This 35-year-old man presented with bilateral shoulder pain. A chordoma of the cervical spine centered around C3-5 was demonstrated on sagittal gadolinium-enhanced $\mathrm{T}_{1}$-weighted MR imaging (Fig. 2 upper left). At an outside institution, the patient underwent a C- 4 and C-5 corpectomy for resection of the tumor followed by anterior reconstruction and plating. Clinically, the patient's symptoms resolved. Approximately 10 months later, the patient experienced a recurrence of symptoms, and follow-up MR imaging revealed recurrence of tumor centered at C2-4 (Fig. 2 upper center). The patient underwent a $\mathrm{C} 2-5$ pos- 


\section{Stereotactic radiosurgery and radiotherapy in chordomas}

terior cervical laminectomy for near-total resection of tumor, followed by lateral mass plating. Two months after the second surgery, the residual chordoma (Fig. 2 upper right) was treated by frameless CyberKnife stereotactic radiosurgery. The residual tumor volume $(1.1 \mathrm{ml})$ was treated with 18.5 Gy to the $75 \%$ isodose line in three fractions of 6.2 Gy to minimize radiation-induced injury to the spinal cord. A secondary collimator measuring $7.5 \mathrm{~mm}$ was used, and the maximum intratumoral dose was 24.7 Gy. Following the second surgery and during the 1-year follow-up period after radiosurgery, the patient continued to be symptom free.

\section{DISCUSSION}

Management of patients with cranial base and cervical chordomas is difficult, as a moderate to high rate of tumor recurrence and poor long-term prognosis have been demonstrated in this population. Historical treatment has generally consisted of tumor resection alone, or resection followed by radiation therapy; however, due to the extensive involvement of the cranial base or spine as well as neurological structures, complete resection is not possible in a significant subset of these patients. ${ }^{11,12,33}$ Furthermore, conventional radiotherapy applied in the treatment of residual or recurrent chordomas has been shown to be of benefit. ${ }^{3,7,8,15,17,22,24,26,29}$ Despite combined surgery and radiotherapy, tumor progression over 5 years has been noted in 24 to $83 \%$ of patients, $3,7,8,15,26$ with 5-year survival ranging from 38 to $79 \%$. $3,15,24,26$

\section{Conventional Radiotherapy}

Conventional radiotherapy has been used to treat chordomas for decades. Debus, et al., ${ }^{7}$ reported using fractionated radiotherapy to treat 45 patients with either postoperative residual chordoma or chondrosarcoma. The mean dose of radiation for chordomas was $66 \mathrm{~Gy}$, and local control was $82 \%$ at 2 years and $50 \%$ at 5 years. Rich, et al. ${ }^{25}$ descibed 48 patients with chordomas who underwent conventional radiotherapy (65-70 Gy) and noted an increased survival rate over those in whom surgery alone was performed. Cummings, et al., ${ }^{6}$ used lower-dose of radiotherapy (40-55 Gy) but still found a survival benefit compared with surgery alone. The authors of several other series have also shown a benefit of radiotherapy in the treatment of chordomas. ${ }^{1,5,9,10,14,19}$

\section{Heavy Charged Particle Radiotherapy}

In using heavy particle irradiation to treat chordomas an attempt is made to take advantage of the steeper falloff dose, which can be better achieved using this modality than with conventional radiotherapy. ${ }^{28} \mathrm{Hug}$, et al., ${ }^{15}$ have reported on 33 patients with chordomas who underwent postresection proton beam radiotherapy. The mean radiation dose was 70.7 Gy (range 64.8-79.2 Gy) and the average follow-up period was 33 months. In 25 (76\%) of the 33 chordomas local control was achieved and the 5-year actuarial survival was $79 \%$. The authors also reported that a smaller treatment volume was associated with a significantly higher rate of chordoma control $(p=0.02)$. Fagundes, et al., ${ }^{8}$ reviewed 204 patients with skull base and cervical chordomas who underwent combined photon- proton radiotherapy at Massachusetts General Hospital over an 18-year period. In $63(31 \%)$ patients tumor progression was observed over a median follow-up period of 54 months. In $60(95 \%)$ of the 63 patients local recurrence was demonstrated within the prior treatment site, leading the authors to conclude that local relapse is the predominant type of treatment failure for skull base chordomas. The authors emphasized the importance of aggressive combined therapy with surgery and irradiation at the time of primary treatment. O'Connell, et al., ${ }^{22}$ studied 62 patients with skull base chordomas treated with proton beam irradiation and concluded, similarly to Hug, et al., that residual tumor volume was a predictor of shortened overall survival following radiotherapy. Raffel, et al., ${ }^{24}$ reviewed 26 patients with cranial chordomas; 23 underwent conventional or heavy particle irradiation or received interstitial implants after resection. Eleven of the 26 patients died (58\% survival) during the 5.6 year average followup period. Benk, et al., ${ }^{3}$ reviewed 18 children in whom photon-proton radiotherapy was used to treat skull base and cervical chordomas at the Massachusetts General Hospital. Using a median dose of $69 \mathrm{cGyE}$ and a 72month median follow up, the 5 year tumor control rate was $63 \%$ and 5-year survival rate was only $68 \%$. The authors also showed that patients with cervical chordoma had a worse survival compared with those harboring cranial base lesions $(\mathrm{p}=0.008)$.

\section{Possible Dose Response}

In several other series authors have studied patients with chordomas treated with varying doses of radiation, and a possible dose response of the radiation was revealed. Romero, et al., ${ }^{26}$ described 18 patients with residual postoperative chordomas who underwent radiotherapy in which a mean dose of 50.12 Gy was used; the overall actuarial 5-year survival was $38 \%$ and the 5-year PFS was $17 \%$. They also showed that the PFS was longer for patients receiving radiation doses greater than $48 \mathrm{~Gy}$ when compared with those receiving doses below $40 \mathrm{~Gy}$. The authors concluded that higher radiation doses increase the disease-free interval. Lybeert and Meerwaldt ${ }^{17}$ reviewed 18 chordoma patients, including four who underwent surgery, four who underwent conventional radiotherapy, and 10 patients who underwent surgery followed by irradiation. Analysis of their results suggested that higher radiation doses result in longer PFS and that the best long-term results can be achieved in patients who undergo surgery followed by radiotherapy. In additional studies some authors have shown that fractionated irradiation is useful in treating patients with chordoma; $;, 3,7,8$ $15,17,22,24,26,29$ however, the potential for toxic doses of radiation to reach critical neurological structures can, in some instances, limit the dose delivered to the tumor itself. ${ }^{32}$

Not all authors believe that there is a dose response when treating chordomas. Catton, et al., ${ }^{4}$ found no significant advantage to be associated with higher radiation doses when treating these tumors, a conclusion also reported by Saxton. ${ }^{27}$ Based on reports of 159 patients with cranial base chordomas reported in the literature, Tai et al., ${ }^{29}$ studied the effects of different treatment modalities. They found that while combined surgery and postoperative irradiation is preferable to either therapy alone with 
respect to patient survival, there was no dose response when using photon irradiation. The authors, however, concluded that methods such as radiosurgery, which would allow increases in dose delivery to the target tumor without increasing morbidity, should increase tumor control.

\section{Stereotactic Radiosurgery}

In a select subgroup of patients with residual or recurrent chordoma stereotactic radiosurgery can be an important treatment option. The steep dose gradient achievable with radiosurgery minimizes radiation outside the tumor target, allowing the delivery of a much larger, and presumably more efficacious, dose to the tumor without exceeding the radiation-related tolerance of normal tissues. Some authors have hypothesized that a therapeutic gain may be achieved by treating slowly proliferating tumors, such as chordomas, with larger radiation doses, which is possible with radiosurgical techniques. ${ }^{31,35}$ In another study the authors showed that better postradiotherapy results were achieved when treating sacral chordomas compared with those of the cervical spine and cranial base, potentially due to the ability to deliver higher doses of radiation to the sacrum. ${ }^{6}$

Muthukumar, et al., ${ }^{21}$ reported on 15 patients (nine with chordoma and six with chondrosarcoma) in whom gamma knife surgery was used as a follow up to an earlier study. ${ }^{16}$ Doses to the tumor margin were between 12 Gy and 20 Gy, and the maximum tumor dose was 24 to 40 Gy. Clinical improvement was demonstrated in eight patients, three remained stable, and four died during the average 4-year follow-up period. Of the surviving 11 patients, the tumor was reduced in size in five, had stabilized in size in five, and had increased in size in one. ${ }^{21}$ Miller, et al., ${ }^{20}$ reviewed the cases of eight patients with skull base chordoma treated with gamma knife surgery. At 2-year follow up, the authors noted a $100 \%$ local control rate and $100 \%$ patient survival rate.

\section{The Stanford Experience}

This study represents our experiences of treating 10 patients with chordomas who underwent LINAC radiosurgery; the average follow-up period was 4 years. From a radiological standpoint, tumor shrinkage or control was observed in $80 \%$ of patients. Despite tumor control in these eight patients, there was no significant improvement in pretreatment symptoms (that is, cranial nerve deficits). No patient developed evidence of radiation-induced necrosis following treatment, including the patient who was treated with two courses of stereotactic radiosurgery, and no other significant treatement-related morbidity was noted. In two patients radiosurgery failed to control tumor size, and one of these patients subsequently underwent repeated radiosurgery after reoperation. These treatment failures may have been caused by inadequate radiation dosage or failure to treat the entire tumor volume due to difficulty in defining tumor margin.

\section{Multimodality Therapy}

Resection is the initial therapy for all patients who are surgical candidates. Postresection, patients are followed clinically and radiographically, and if there is evidence of tumor recurrence, patients are considered candidates for stereotactic radiosurgery. Most patients (seven) in this series had undergone at least two prior operations, and they had sustained significant clinical deficits due to the presence of recurrent tumor as well as surgery-related morbidity. For these patients, stereotactic radiosurgery represented a noninvasive method for treating their recurrent/residual tumors. Multimodality treatment-resection followed by radiosurgery for postoperative residual chordomas - may result in a lower morbidity rate than that for patients undergoing multiple surgical procedures. It is not clear at the present time whether resection followed by radiosurgery represents a clear survival advantage over surgery followed by conventional radiotherapy.

\section{Role of Repeated Surgical Resection}

Not all patients with postresection tumor remnants or tumor growth are candidates for radiosurgery. As with other tumors considered for radiosurgery, chordomas larger than 3 to $3.5 \mathrm{~cm}$ in maximum diameter are difficult to treat without increasing radiation-related risks to the patient. Smaller-sized chordomas treated by radiosurgery may also represent a high risk if they are located adjacent to critical structures, such as the anterior visual pathways. Although the frameless CyberKnife system allows increased flexibility with respect to fractionated radiosurgical regimens, such fractionation may reduce radiationrelated efficacy on the tumor. In some of these patients, repeated resection may produce more favorable results than radiosurgery. Furthermore, patients with significant chordoma-induced mass effect should not undergo radiosurgery as the likelihood of significant reduction in tumor volume following radiosurgery treatment is low.

\section{Potential Drawbacks of Radiosurgery}

Despite the theoretical benefits of using radiosurgery to treat chordomas, there are obvious drawbacks as well. Radiosurgery, by definition, requires precise target delineation. Although neuroimaging techniques are much improved, the anatomy of the skull base is especially complex. One may question whether modern imaging can reliably vizualize the full extent of a tumor in this location, as microscopic rests of tumor may not be visible even on the best-quality MR images. Such concerns are of less importance in open resection and radiotherapy. Furthermore, despite the focused nature of radiosurgery, the close spatial relationship between many chordomas and critical brain structures inevitably results in some irradiation of nontumor tissue. Given the limited dose tolerance of many base-of-brain critical structures and the discreet spatial inaccuracies inherent in stereotactic frames, ${ }^{18}$ the possibility of damaging normal neurological tissue remains a risk. Additionally, given the potential slow-growing course of some chordomas, which is probably best discussed in terms of years and not months, the primary weakness of this and other published radiosurgery reports on chordomas is the relatively short follow-up period. Longer follow-up review of these patients is necessary before we can provide estimates on 5- and 10-year PFS and be certain that such tumors are completely ablated. 


\section{Stereotactic radiosurgery and radiotherapy in chordomas}

\section{CONCLUSIONS}

Stereotactic radiosurgery appears to be an effective means to treat residual or recurrent chordomas following prior resection. Although our reported follow up is not as long as that documented in some series of fractionated radiotherapy and heavy charged particle irradiation, radiographically demonstrated control rates for cranial base and cervical chordomas appear to be at least comparable, if not superior, to those for photon or proton beam radiotherapy. Because radiosurgery is an outpatient procedure, minimally invasive, well tolerated, and in most cases, cost effective relative to repeated surgery, it represents a possible option for the treatment of patients with residual or recurrent chordoma following prior resection.

\section{Acknowledgment}

We thank Beth Houle for her assistance with figure production.

\section{References}

1. Amendola BE, Amendola MA, Oliver E, et al: Chordoma: role of radiation therapy. Radiology 158:839-843, 1986

2. Austin-Seymour M, Munzenrider J, Goitein M, et al: Fractionated proton radiation therapy of chordoma and low-grade chondrosarcoma of the base of the skull. J Neurosurg 70:13-17, 1989

3. Benk V, Liebsch NJ, Munzenrider JE, et al: Base of skull and cervical spine chordomas in children treated by high-dose irradiation. Int J Radiat Oncol Biol Phys 31:577-581, 1995

4. Catton C, O'Sullivan B, Bell R, et al: Chordoma: long-term follow-up after radical photon irradiation. Radiother Oncol 41: 67-72, 1996

5. Chetiyawardana AD: Chordoma: results of treatment. Clin Radiol 35:159-161, 1984

6. Cummings BJ, Hodson DI, Bush RS: Chordoma: the results of megavoltage radiation therapy. Int J Radiat Oncol Biol Phys 9:633-642, 1983

7. Debus J, Schulz-Ertner D, Schad L, et al: Stereotactic fractionated radiotherapy for chordomas and chondrosarcomas of the skull base. Int J Radiat Oncol Biol Phys 47:591-596, 2000

8. Fagundes MA, Hug EB, Liebsch NJ, et al: Radiation therapy for chordomas of the base of skull and cervical spine: patterns of failure and outcome after relapse. Int J Radiat Oncol Biol Phys 33:579-584, 1995

9. Forsyth PA, Cascino TL, Shaw EG, et al: Intracranial chordomas: a clinicopathological and prognostic study of 51 cases. $\mathbf{J}$ Neurosurg 78:741-747, 1993

10. Fuller DB, Bloom JG: Radiotherapy for chordoma. Int J Radiat Oncol Biol Phys 15:331-339, 1988

11. Gay E, Sekhar LN, Rubinstein E, et al: Chordomas and chondrosarcomas of the cranial base: results and follow-up of $60 \mathrm{pa}-$ tients. Neurosurgery 36:887-897, 1995

12. Gay E, Sekhar LN, Wright DC: Chordomas and chondrosarcomas of the cranial base, in Kaye AH, Laws ER Jr (eds): Brain Tumors: An Encyclopedic Approach. Edinburgh: Churchill Livingstone, 1995, pp 777-794

13. Gehan EA, Tefft MC: Will there be resistance to the RECIST (Response Evaluation Criteria in Solid Tumors)? J Natl Cancer Inst 92:179-181, 2000

14. Heffelfinger MJ, Dahlin DC, MacCarty CS, et al: Chordomas and cartilaginous tumors at the skull base. Cancer 32:410-420, 1973

15. Hug EB, Loredo LN, Slater JD, et al: Proton radiation therapy for chordomas and chondrosarcomas of the skull base. J Neurosurg 91:432-439, 1999
16. Kondziolka D, Lunsford LD, Flickinger JC: The role of radiosurgery in the management of chordoma and chondrosarcoma of the cranial base. Neurosurgery 29:38-46, 1991

17. Lybeert ML, Meerwaldt JH: Chordoma. Report on treatment results in eighteen cases. Acta Radiol Oncol 25:41-43, 1986

18. Maciunas RJ, Galloway RL, Latimer JW: The application accuracy of stereotactic frames. Neurosurgery 35:682-695, 1994

19. Magrini SM, Papi MG, Marletta F, et al: Chordoma-natural history, treatment and prognosis. The Florence Radiotherapy Department experience (1956-1990) and a critical review of the literature. Acta Oncol 31:847-851, 1992

20. Miller RC, Foote RL, Coffey RJ, et al: The role of stereotactic radiosurgery in the treatment of malignant skull base tumors. Int J Radiat Oncol Biol Phys 39:977-981, 1997

21. Muthukumar N, Kondziolka D, Lunsford LD, et al: Stereotactic radiosurgery for chordoma and chondrosarcoma: further experiences. Int J Radiat Oncol Biol Phys 41:387-392, 1998

22. O'Connell JX, Renard LG, Liebsch NJ, et al: Base of skull chordoma. A correlative study of histologic and clinical features of 62 cases. Cancer 74:2261-2267, 1994

23. Okazaki H, Scheithauer BW: Atlas of Neuropathology. New York: Gower Medical Publishing, 1988

24. Raffel C, Wright DC, Gutin PH, et al: Cranial chordomas: clinical presentation and results of operative and radiation therapy in twenty-six patients. Neurosurgery 17:703-710, 1985

25. Rich TA, Schiller A, Suit HD, et al: Clinical and pathologic review of 48 cases of chordoma. Cancer 56:182-187, 1985

26. Romero J, Cardenes H, la Torre A, et al: Chordoma: results of radiation therapy in eighteen patients. Radiother Oncol 29: 27-32, 1993

27. Saxton JP: Chordoma. Int J Radiat Oncol Biol Phys 7: 913-915, 1981

28. Suit HD, Goitein M, Munzenrider J, et al: Definitive radiation therapy for chordoma and chondrosarcoma of base of skull and cervical spine. J Neurosurg 56:377-385, 1982

29. Tai PT, Craighead P, Bagdon F: Optimization of radiotherapy for patients with cranial chordoma. A review of dose-response ratios for photon techniques. Cancer 75:749-756, 1995

30. Thames HD, Withers HR, Peters LJ, et al: Changes in early and late radiation responses with altered dose fractionation: implications for dose-survival relationships. Int J Radiat Oncol Biol Phys 8:219-226, 1982

31. Therasse P, Arbuck SG, Eisenhauer EA, et al: New guidelines to evaluate the response to treatment in solid tumors. J Natl Cancer Inst 92:205-216, 2000

32. Urie MM, Fullerton B, Tatsuzaki $\mathrm{H}$, et al: A dose response analysis of injury to cranial nerves and/or nuclei following proton beam radiation therapy. Int J Radiat Oncol Biol Phys 23: 27-39, 1992

33. Watkins L, Khudados ES, Kaleoglu M, et al: Skull base chordomas: a review of 38 patients, 1958-1988. Br J Neurosurg 7:241-248, 1993

34. Winston KR, Lutz W: Linear accelerator as a neurosurgical tool for stereotactic radiosurgery. Neurosurgery 22:454-464, 1988

35. Withers HR, Thames HD, Peters LJ: Biological bases for high RBE values for late effects of neutron irradiation. Int J Radiat Oncol Biol Phys 8:2071-2076, 1982

Manuscript received January 16, 2001.

Accepted in final form February 15, 2001.

This work was supported by the Alice and Stanley Schlicktman, the Arillaga Family, and the Chen Family Gift funds to John R. Adler, Jr., M.D.

Address reprint requests to: Steven D. Chang, M.D., Department of Neurosurgery, Room R-291, Stanford University Medical Center, 300 Pasteur Drive, Stanford, California 94305. email: sdchang @ stanford.edu. 